ORIGINAL ARTICLE

\title{
Desmoplasia measured by computer assisted image analysis: an independent prognostic marker in colorectal carcinoma
}

\author{
B Sis, S Sarioglu, S Sokmen, M Sakar, A Kupelioglu, M Fuzun
}

J Clin Pathol 2005;58:32-38. doi: 10.1136/icp.2004.018705

See end of article for authors' affiliations

.....................

Correspondence to: Dr S Sarioglu, Dokuz Eylül University School of Medicine, Department of Pathology, Inciraltı, Izmir 35340, Turkey; sulen. sarioglu@deu.edu.tr

Accepted for publication 27 July 2004
Aims: The assessment of desmoplasia by traditional semiquantitative methods does not provide reliable prognostic data. The aim of this study was to quantify desmoplasia by computerised image analysis in primary colorectal carcinomas and to investigate its ability to predict overall survival.

Methods: In total, 112 colorectal adenocarcinomas, with a median follow up of 66 months, were studied. The representative tumour sections were stained by the van Gieson method, which stains collagen rich stroma red. For quantitative histochemical measurement, digital images were analysed by a computerised image analysis program to calculate the percentage of red stained tissue area. The percentage of desmoplasia (PD) was related to conventional clinicopathological prognostic factors and overall survival. Results: The mean (SD) PD was 4.85 (3.37). PD was found to be significantly associated with lymph vessel and venous invasion. By Kaplan-Meier analysis, PD was associated with survival-patients with PD $>4$ had a shorter survival than those with $P D \leqslant 4$. In multivariate analysis, tumour stage, distant metastasis, and PD emerged as independent prognostic factors.

Conclusion: Desmoplasia measured by image analysis seems to be a significant prognostic indicator in patients with colorectal carcinoma and the improved method described in this study would be useful for routine prognostication.
$\mathrm{D}$ uring epithelial carcinogenesis, it is not only the epithelial cells that change, but the stroma also. The morphological signs of cancer associated stromal alterations are desmoplasia, angiogenesis, and inflammatory cell infiltration. ${ }^{12}$ Cancer cell invasion usually induces new extracellular matrix formation and the activation of fibroblast-like cells, known as the desmoplastic reaction. ${ }^{1-3}$ This desmoplastic reaction is first observed with the invasive phenotype, and is not seen in carcinoma in situ or benign tumours. ${ }^{2}$ Some invasive tumours trigger a severe desmoplastic response characterised by abundant stroma rich in collagen. ${ }^{3}$

\section{"We have devised an objective and simple method to measure desmoplasia in colorectal carcinomas by com- puter assisted image analysis"}

The biological relevance of the desmoplastic host reaction is not fully understood. Initially, it was suggested that this response could play a protective role and limit the process of tumour invasion. ${ }^{3}$ However, recent evidence suggests that molecular crosstalk between neoplastic cells and stromal cells, and cancer induced changes in the stroma, modify the differentiation, proliferative capacity, and invasive capacity of tumour cells. ${ }^{1-6}$ In a xenograft model, injection of human prostate cancer cells combined with stromal components into athymic nude mice resulted in a high tumour incidence as a result of the stimulation of early angiogenesis and tumour growth, whereas prostate cancer cells alone were essentially non-tumorigenic. ${ }^{5}$ In another experimental study, the transplantation of colon carcinoma cells into reactive subcutaneous granulation tissue induced invasive colon carcinomas, but transplantation of colon carcinoma cells into undisturbed subcutaneous connective tissue resulted in the formation of encapsulated, non-invasive, well differentiated tumours. ${ }^{6}$
A variety of clinical studies of human lung and breast carcinomas and squamous cell carcinomas of the skin confirmed these experimental data, and suggested an association between desmoplasia and tumour invasion, development of metastases, or recurrence. ${ }^{7-9}$ In colon cancer, studies of desmoplasia gave more conflicting results. A small number of studies suggested that a peritumorous stromal response was an adverse prognostic factor. ${ }^{10}{ }^{11}$ However, other studies of desmoplasia have failed to demonstrate prognostic significance for this factor. ${ }^{10}$ These discrepancies seem to be mostly the result of technical errors, such as subjective grading systems (including semiquantitative methods), interobserver and intraobserver variation, or sampling error. The case volume is also an important factor. The amount of desmoplasia in colorectal carcinoma was traditionally graded as: little, moderate, and extensive. ${ }^{12}$ Such a semiquantitative grading system is easy to use but seems to be unreliable. The College of American Pathologists recommended that desmoplasia should be evaluated by a uniform method as an individual variable in large studies on prognostic factors using multivariate analysis. ${ }^{12}$

To date, no quantitative measures have been used to assess desmoplasia in colorectal carcinomas, perhaps because of its unknown biological relevance. Hence, we have devised an objective and simple method to measure desmoplasia in colorectal carcinomas by computer assisted image analysis. The purpose of our study was to evaluate the prognostic relevance of desmoplasia in primary colorectal carcinomas using this novel method.

\section{MATERIALS AND METHODS}

Tissue sections were cut from the archival, paraffin wax embedded resection specimens of 112 patients with primary

Abbreviations: CV, coefficient of variation; PD, percentage of desmoplasia 


\begin{tabular}{|c|c|c|c|}
\hline Features & $\mathrm{N}(\%)$ & Mean (SD) PD & $\mathrm{p}$ Value \\
\hline Total & 112 & & \\
\hline \multicolumn{4}{|l|}{ Age } \\
\hline \multicolumn{4}{|c|}{$\begin{array}{l}\text { Mean (SD): } 61.2 \text { (13.6) years } \\
\text { Range: } 24-95\end{array}$} \\
\hline MAC stage & & & 0.12 \\
\hline B1 & $11(9.8)$ & $4.09(2.34)$ & \\
\hline B2 & $52(46.4)$ & 4.38 (3.03) & \\
\hline $\mathrm{Cl}$ & $3(2.7)$ & $4.33(3.51$ & \\
\hline C2 & $36(32.1)$ & $5.50\lceil 3.73$ & \\
\hline D & $10(8.9)$ & $6.0(4.52)$ & \\
\hline \multicolumn{3}{|l|}{ AJCC group } & 0.12 \\
\hline Stage I & $11(9.8)$ & $4.09(2.34)$ & \\
\hline Stage IIA & $52(46.4)$ & $4.38(3.03)$ & \\
\hline Stage IIIA & $3(2.7)$ & $4.33(3.51)$ & \\
\hline Stage IIIB & $36(32.1)$ & $5.50(3.73)$ & \\
\hline Stage IV & $10(8.9)$ & $6.0(4.52)$ & \\
\hline \multicolumn{3}{|l|}{ Lymph node involvement } & 0.06 \\
\hline Negative & $65(58)$ & $4.43(3.07)$ & \\
\hline$<4$ & $30(27)$ & $5.13(4.18)$ & \\
\hline$\geqslant 4$ & $17(15)$ & $6.0(2.71)$ & \\
\hline \multicolumn{3}{|l|}{ Distant metastasis } & 0.51 \\
\hline Negative & $102(91)$ & $4.74(3.25)$ & \\
\hline Positive & $10(8.9)$ & $6.0(4.52)$ & \\
\hline \multicolumn{3}{|l|}{ Venous invasion } & 0.02 \\
\hline Negative & $98(87)$ & $4.63(3.37)$ & \\
\hline Positive & $14(12)$ & $6.42(3.10)$ & \\
\hline \multicolumn{3}{|l|}{ Lymphatic invasion } & 0.01 \\
\hline Negative & $73(65)$ & $4.32(3.24)$ & \\
\hline Positive & $39(35)$ & $5.84(3.43)$ & \\
\hline \multicolumn{3}{|l|}{ Perineural invasion } & 0.11 \\
\hline Negative & $75(67)$ & $4.70(3.68)$ & \\
\hline Positive & $37(33)$ & $5.16(2.66)$ & \\
\hline \multicolumn{3}{|l|}{ Tumour grade } & 0.22 \\
\hline Well differentiated & 20 (18) & $4.45(2.76)$ & \\
\hline Moderately differentiated & $63(56)$ & $5.52(3.77)$ & \\
\hline Poorly differentiated & $29(26)$ & $3.68(2.46)$ & \\
\hline
\end{tabular}

colorectal adenocarcinoma treated between 1991 and 1997. The clinical records of all patients were reviewed for age, sex, primary tumour site, tumour size, and the occurrence of distant metastasis. Haematoxylin and eosin stained sections of the specimens were reviewed by one investigator (BS) and conventional histopathological prognostic parameters in colorectal carcinoma, such as tumour grade, depth of invasion, lymph node involvement, and vascular and perineural invasion, were noted. Conventional semiquantitative grading for desmoplasia ${ }^{12}$ was also carried out. The tumours were staged according to the modified Astler-Coller staging system, ${ }^{13}$ and graded according to the World Health Organisation criteria. ${ }^{14}$ The clinical stage of disease was determined according to the AJCC staging system. ${ }^{15}$ Table 1 details the distribution of clinicopathological variables in the patients. All patients were followed up until death or for a median of 66 months (range, 48-120). During follow up, 38
(33.9\%) patients died-37 as a result of distant metastasis and one as a result of postoperative complications. The median survival time was 42.5 months (range, 1-108). Twelve $(10.7 \%)$ patients were out of follow up.

\section{Staining}

The most representative paraffin wax block from each tumour was chosen and cut into $5 \mu \mathrm{m}$ thick sections. The sections were dewaxed, rehydrated, and then stained with van Gieson's solution (saturated aqueous picric acid and $1 \%$ aqueous acid fuchsin) to demonstrate collagen rich stroma. ${ }^{16}$

\section{Semiquantitative scoring}

For each case, the section stained by van Gieson's method was observed by light microscopy and scored semiquantitatively for desmoplasia as: little, moderate, and extensive.
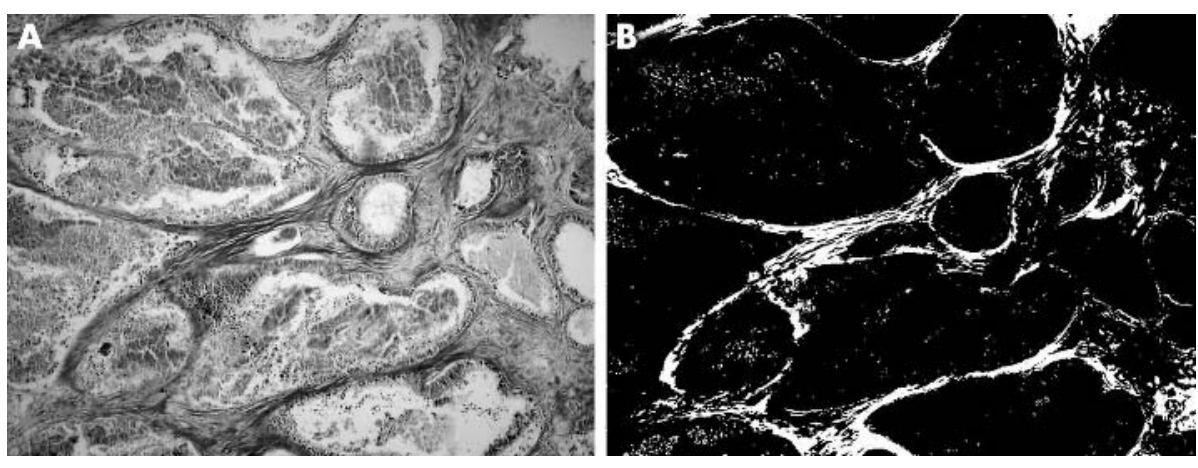

Figure 1 Section of colorectal carcinoma stained with van Gieson (A) before and (B) after the selection of red colour. 


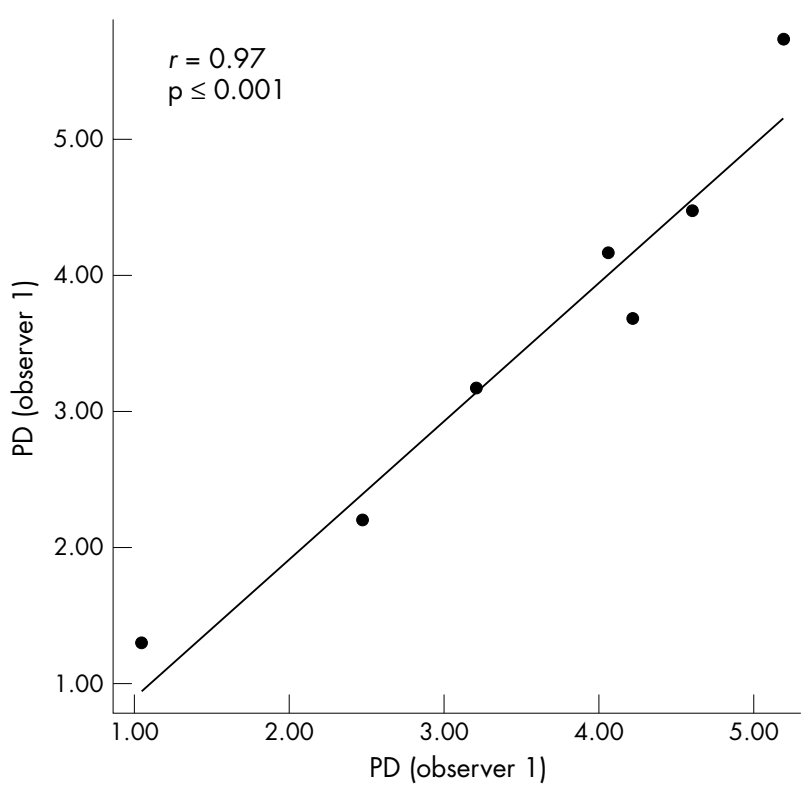

Figure 2 Intraobserver agreement on the measurement of percentage of desmoplasia (PD). Test statistics: Spearman's rank correlation test, $r=0.97, p \leqslant 0.001$.

\section{Image analysis}

Digital images obtained from the van Gieson stained tumour sections using a light microscope (Labophot-2; Nikon, Tokyo, Japan) at $\times 4$ magnification and a digital colour video camera (WK-C220E; Hitachi, Tokyo, Japan) with image analysis software (Mediscope; Dokuz Eylul University Clinic Engineering Department, Izmir, Turkey) were collected and stored on a computer (Vestel, Manisa, Turkey). In each case, 10 digital images (total area, $26.1 \mathrm{~mm}^{2}$ ) were selected and analysed by automatic processing.

The software was intended for colour segmentation including a determined neighbourhood of colours. The colour selected by the pathologist is accepted as the index for automatic choosing of the stained area.

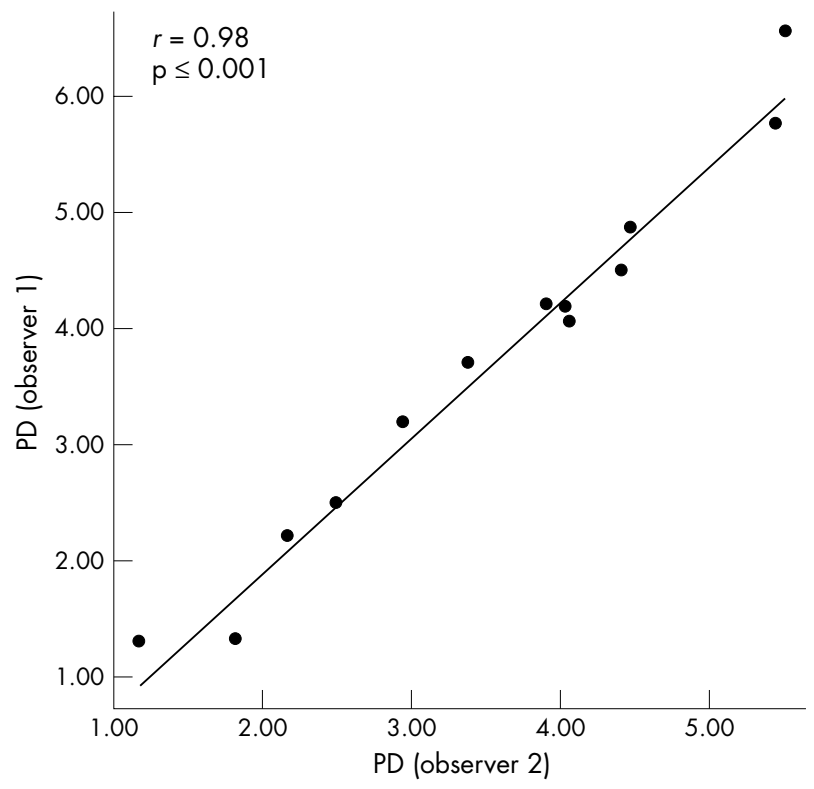

Figure 3 Interobserver agreement on the measurement of percentage of desmoplasia (PD). Test statistics: Spearman's rank correlation test, $r=0.98, p \leqslant 0.001$.
For each captured area, the percentage of red stained area was determined semiautomatically. First, the red stained area was selected by the pathologist using visual observation by multiple clicking on the image. Subsequently, the system selected the areas with the same degree and configuration of staining, converted into pixel density units, and presented the area of selection as a percentage within each field. To allow the pathologist to repeat the procedure until the optimum selection was achieved, each selected view could be examined by the pathologist. The mean of 10 fields was determined and called the percentage of desmoplastic stroma (fig 1A, B), and the percentage of desmoplasia (PD) was determined by dividing the selected area by the total test area. ${ }^{17} 18$

\section{Reproducibility analysis}

One representative van Gieson stained tumour section of 13 resection specimens was measured separately by two observers, and seven specimens were measured twice at intervals by one observer. Next, we determined the interobserver variation, intraobserver variation, and coefficient of variation (CV (SD/mean)). ${ }^{19-21}$ To test the reproducibility, we also used scatter plots and calculated Spearman's rank correlation coefficient $(r)$. In addition, we also performed a reliability analysis by calculating the coefficient of intraclass correlation using ANOVA. ${ }^{19}$

\section{Statistical analysis}

Statistical analysis was performed using computer software (SPSS 12.0; SPSS, Chicago, Illinois, USA). A probability level of 0.05 or less was chosen to represent significance. Differences between groups were analysed with the Pearson $\chi^{2}$ test, one way ANOVA, and independent samples $t$ tests. The relation between variables was investigated using Spearman's rank correlation and Kendall's tau-B correlation tests. Univariate survival analyses were performed with the Kaplan-Meier method and multivariate analyses with the Cox proportional hazards model. For Kaplan-Meier, comparisons among groups were carried out using the log rank test.

\section{RESULTS}

\section{Reproducibility analysis of the measurement of desmoplasia}

Table 2 shows the mean PD variance and CV for interobserver and intraobserver reproducibility studies. The CV of PD was 0.73 for intraobserver variation and 1.1 for interobserver variation. Reproducibility is equal to $100-\mathrm{CV} .{ }^{21}$ As shown in table 2, the coefficient of intraclass correlation of 0.98 of both intra-assay and interassay studies showed that the measurement of desmoplasia by image analysis was a highly reliable method, and similar results of reliability analysis for computer assisted measurement of fibrosis have been obtained before. ${ }^{19}$ Spearman's rank correlation coefficients

Table 2 Reproducibility and reliability studies for the measurement of desmoplasia by computer assisted image analysis

\begin{tabular}{lll}
\hline & Interobserver variation & Intraobserver variation \\
\hline Mean & 0.2431 & 0.2714 \\
$\mathrm{SD}$ & 0.2719 & 0.2006 \\
$\mathrm{SE}$ & 0.0754 & 0.0758 \\
Variance & 0.074 & 0.04 \\
$\mathrm{CV}$ & 1.1 & 0.73 \\
& Intraclass correlation & $95 \% \mathrm{Cl}$ \\
Interobserver study & 0.986 & $0.955-0.996$ \\
Intraobserver study & 0.985 & $0.913-0.997$ \\
\hline \multirow{2}{*}{$\mathrm{Cl}$, confidence interval; CV, coefficient of variance. }
\end{tabular}




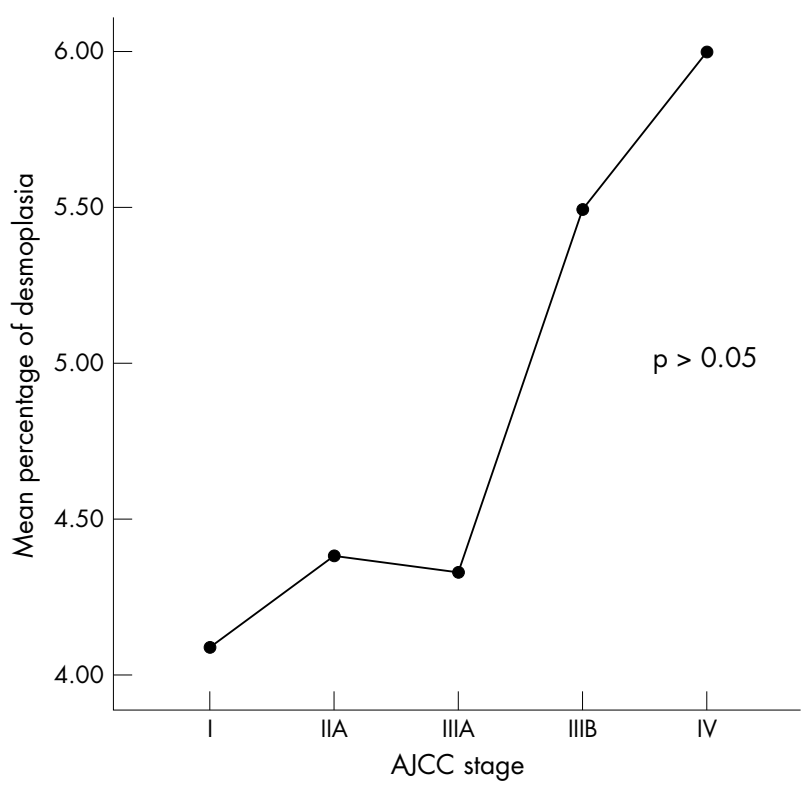

Figure 4 Mean percentage of desmoplasia in tumours in relation to clinical stage of disease.

also showed a substantial intraobserver $(r=0.97)$ and interobserver $(r=0.98)$ agreement (figs 2 and 3).

\section{Percentage of desmoplasia}

The mean PD was 4.85 (SD, 3.37; range, 1-18). The cases were also grouped according to the median value (4.0): PD $\leqslant 4($ group 1$)$ and $>4($ group 2$)$.

Table 1 showed the relation between $\mathrm{PD}$ and the clinicopathological parameters. The mean (SD) PD of patients with stage I disease was $4.09(2.34)$ and for stage IV patients this increased to 6.0 (4.52); however, the difference was not significant $(p>0.05$; table 1 ; fig 4$)$. PD was found to be

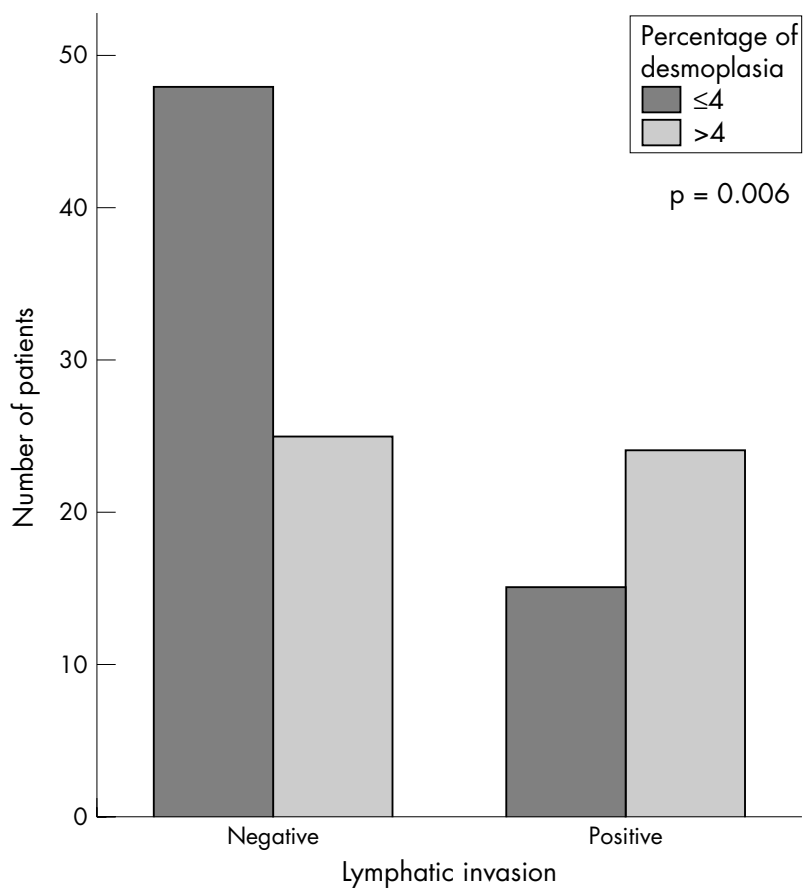

Figure 5 The distribution of lymphatic invasion in tumours according to the percentage of desmoplasia. significantly associated with lymph vessel and venous invasion ( $\mathrm{p}=0.006$ and $\mathrm{p}=0.02$, respectively; figs 5 and $6)$. No significant associations were found between PD and tumour grade, perineural invasion, lymph node involvement, or distant metastasis $(\mathrm{p}>0.05)$.

Using the semiquantitative method, $11(10 \%)$ cases had little desmoplasia, 88 (78\%) moderate, and 13 (11\%) extensive. No significant relations were found between semiquantitative desmoplasia values and other clinicopathological parameters $(\mathrm{p}>0.05)$. Table 3 provides a comparison of the desmoplasia values obtained by quantitative image analysis and the semiquantitative method. PD values were not significantly different in cases with different desmoplasia grades $(p>0.05)$. The median PD was similar among the different grades (fig 7).

In univariate survival analysis, tumour stage $(p \leqslant 0.001)$, distant metastasis $(p \leqslant 0.001)$, venous invasion $(p=$ $0.006)$, perineural invasion $(p=0.001)$, semiquantitative desmoplasia values $(\mathrm{p}=0.03)$, and PD $(\mathrm{p}=0.008)$ were found to be significantly related to survival (table 4; fig 8 ). Multivariate analysis showed tumour stage $(p \leqslant 0.001)$, distant metastasis $(p \leqslant 0.001)$, and PD $(p=0.01)$ to be independent determinants of survival.

\section{DISCUSSION}

The biological relevance of the desmoplastic response to cancer is still unclear. Initially, it was suggested to act as a barrier and inhibit tumour invasion. ${ }^{3}$ Inhibition of the desmoplastic response in mice has been reported to increase the invasion and metastasis of the BL6 melanoma cell line. ${ }^{22}$ Hewitt and colleagues ${ }^{3}$ found little evidence of a desmoplastic response at the invasive edge of colorectal carcinomas-there was limited collagen I immunostaining and sparsely distributed or absent myofibroblasts-and suggested that desmoplasia fails to prevent cancer invasion because it is lacking at the invasive edge. It has also been proposed that myofibroblasts around in situ ductal breast carcinoma prevent cancer invasion by stabilisation of the peritumorous matrix through lysyl oxidase secretion. ${ }^{23}$

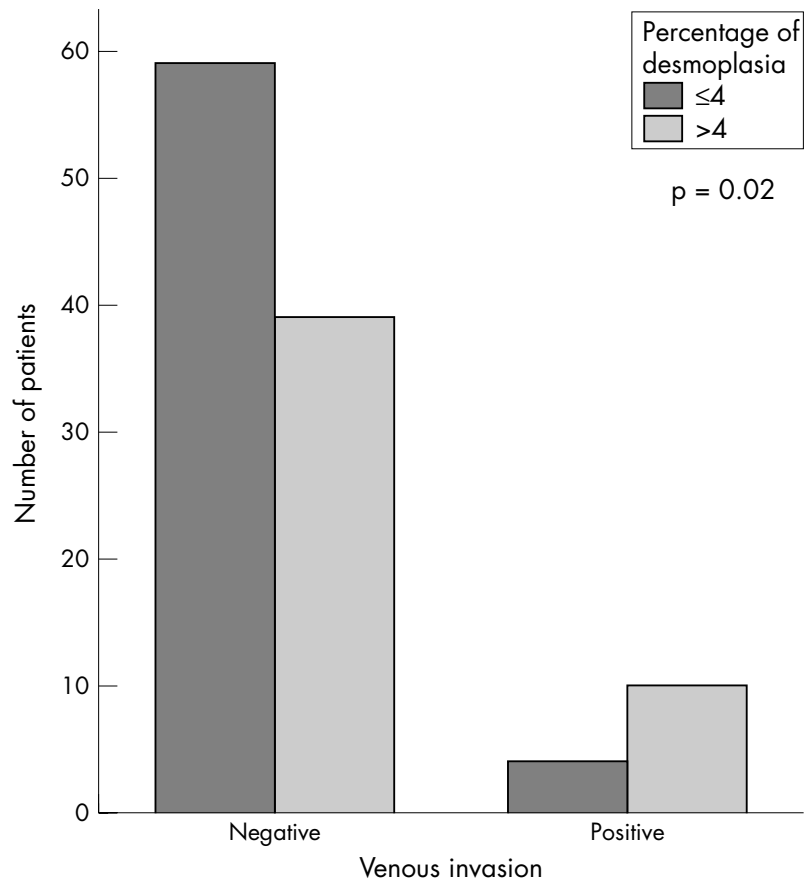

Figure 6 The distribution of venous invasion in tumours according to the percentage of desmoplasia. 


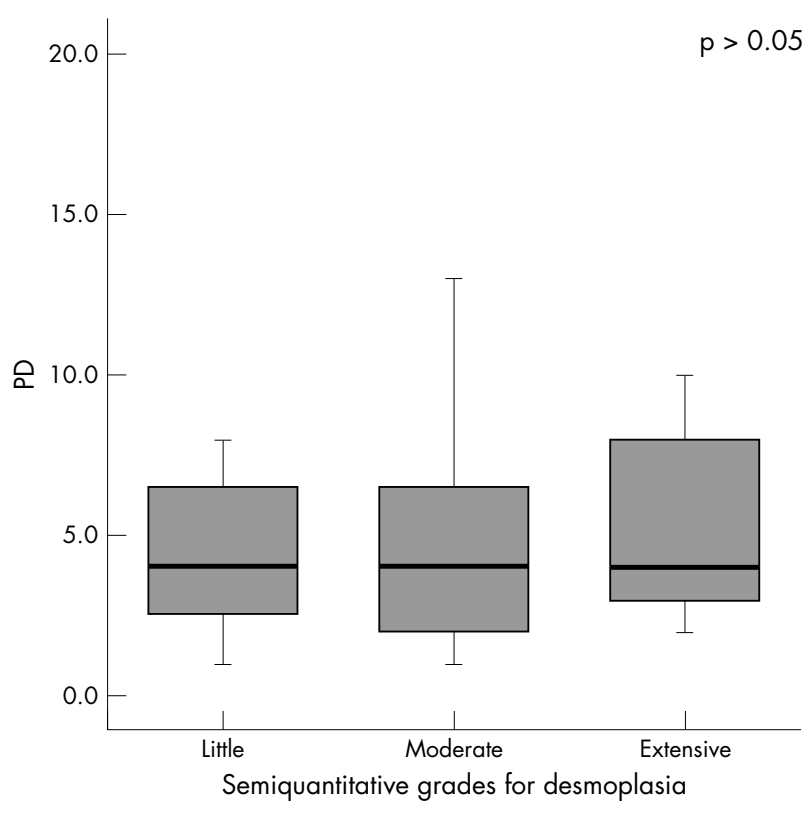

Figure 7 Variation in the percentage of desmoplasia (PD) at each value of the semiquantitative grades for desmoplasia.

In contrast, there is growing evidence that the stromal reaction may play an important role in triggering cancer invasion. ${ }^{24}{ }^{25}$ As reviewed by Ohtani, ${ }^{2}$ cancer invasion usually induces new extracellular matrix formation by the activation of stromal cells, which is a desmoplastic reaction, and this process includes both matrix production and degradation. It is already known that cancer stroma resembles granulation tissue because of the presence of myofibroblasts and active matrix degrading processes. ${ }^{126}$ Degradation of extracellular matrix plays a crucial role in tumour invasion and metastasis, and matrix degrading proteases, such as matrix metalloproteinases, are synthesised mainly by the stromal cells of cancer tissue. $^{26}$

An interesting experimental study by Dingemans and colleagues $^{6}$ showed that the transplantation of colon carcinoma cells into reactive subcutaneous granulation tissue induced invasive colon carcinomas, whereas transplantation into undisturbed subcutaneous connective tissue formed encapsulated, non-invasive, and well differentiated tumours. As reviewed by De Wever and Mareel $^{1}$ and Bosman et al, ${ }^{27}$ the molecular crosstalk between cancer cells and the stroma may contribute to cancer invasion and may modulate differentiation in cancer cells. Martin et al proposed that stromal myofibroblasts alter the migratory properties of colon cancer cells and play a role in the invasion and metastasis of colorectal cancer. ${ }^{28}$ Similarly, Dimanche-Boitrel et al showed that stromal cells, more specifically myofibroblasts, contribute to the invasiveness of a rat colon cancer cell line in vivo and in vitro. ${ }^{29}$ In vitro experiments in rat colorectal tumour models by Lieubeau and colleagues ${ }^{30}$ showed that tumour

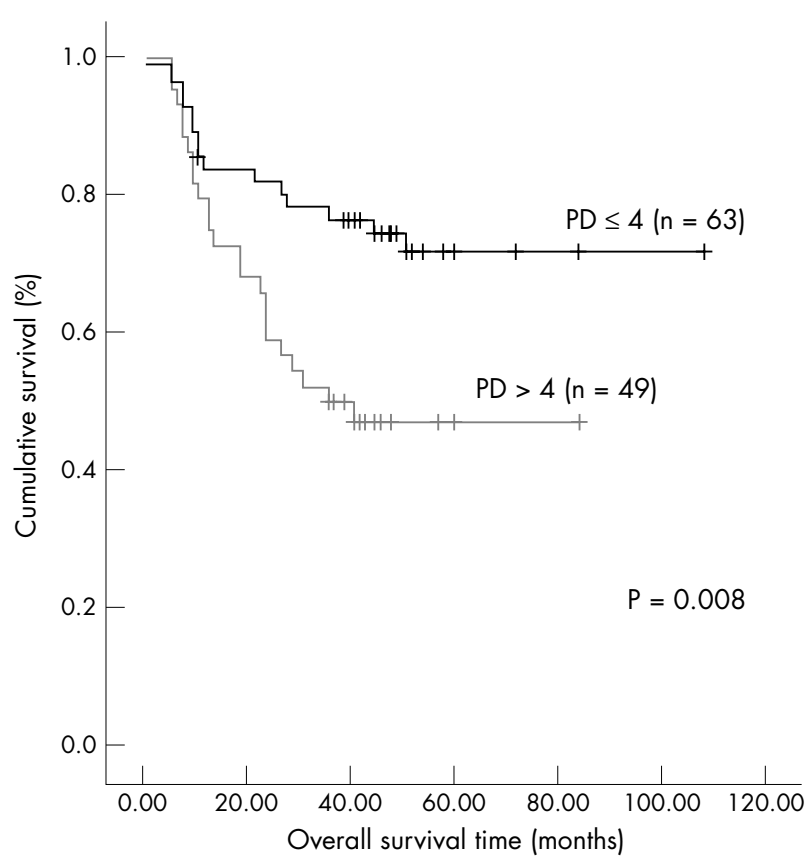

Figure 8 Kaplan-Meier overall survival curves for 112 patients with colorectal carcinoma grouped according to their percentage of desmoplasia (PD). Test statistics: log rank test (one degree of freedom), $\mathrm{p}=0.008$.

activated myofibroblasts prevent the penetration of $\mathrm{T}$ cells and macrophages within tumour nodules, and they suggested that successful immunotherapeutic strategies should include complementary treatments against tumour associated myofibroblasts.

A few clinical studies point out an interesting association between intratumorous hypoxia and the presence of fibrosis in human malignant tumours, particularly in breast cancer. $^{31}{ }^{32}$ Intratumorous hypoxia significantly correlates with tumour invasion, metastasis, and poor prognosis in several malignant tumours, and it is also the driving force behind angiogenesis. ${ }^{31-35}$ Colpaert et al reported the presence of fibrotic foci in breast cancer as a marker of intratumorous hypoxia and hypoxia driven angiogenesis, and showed that it was associated with a worse survival. ${ }^{31}{ }^{32}$

Despite the growing experimental evidence of the biological importance of tumour stroma, both in vitro and in vivo, a small number of clinical studies have shown the prognostic value of desmoplasia in colorectal carcinoma. ${ }^{10}$ These conflicting data are probably attributable to the small number of cases, the interobserver and intraobserver variation in semiquantitative scoring systems, and/or sampling variation.

In our present study, quantitative histochemical analysis of collagenous stroma (desmoplasia) was performed using van Gieson's staining method, which stains collagen fibres red. Other collagen binding stains, such as picro-Sirius red or

Table 3 Variation in percentage of desmoplasia (PD) at each value of the semiquantitative grades for desmoplasia in 112 primary colorectal carcinomas

\begin{tabular}{|c|c|c|c|c|c|}
\hline \multirow{2}{*}{$\begin{array}{l}\text { Semiquantitative grades for } \\
\text { desmoplasia }\end{array}$} & \multirow[b]{2}{*}{$\mathbf{N}(\%)$} & \multicolumn{2}{|l|}{ PD } & \multirow[b]{2}{*}{ p Value* } & \multirow[b]{2}{*}{$r$ Value } \\
\hline & & Mean (SD) & Median & & \\
\hline Little & $11(10)$ & $4.27(2.61)$ & 4 & 0.35 & 0.09 \\
\hline Moderate & $88(78)$ & 4.75 (3.28) & 4 & & \\
\hline Extensive & $13(11)$ & $6.07(4.42)$ & 4 & & \\
\hline
\end{tabular}

*By means of ANOVA; †Spearman's rank correlation coefficient. 
Table 4 Survival rates and mean survival time according to the percentage of desmoplasia (PD)

\begin{tabular}{lllll}
\hline PD & $\mathbf{N}(\%)$ & Survival rate $(\%)$ & $\begin{array}{l}\text { Mean survival time } \\
\text { (months) }\end{array}$ & p Value \\
\hline$\leqslant 4$ & $63(56)$ & 56.3 & $83.34(5.49)$ & 0.008 \\
$>4$ & $49(44)$ & 43.8 & $49.24(5.11)$ & \\
\hline
\end{tabular}

Masson's trichrome stain, could be used with this computer assisted quantification method. We chose the van Gieson method because it is commonly used in most routine pathology laboratories, it is very easy to apply, and it does not need polarising microscopy.

"Only the measurement of desmoplasia by the quantitative method retained a clear independent association with overall survival" $^{\prime \prime}$

Different morphometric methods have been described for the measurement of fibrosis, particularly in kidney and liver disease. ${ }^{19-21}{ }^{36}$ In our current study, we measured the amount of desmoplasia in 112 primary colorectal carcinomas using computer assisted image analysis. The method was easy to perform, although it is more laborious and time consuming than semiquantitative techniques, and requires more complex equipment, including a digital capture system, computer, and software. However, it is the preferred method because it is reliable and allows desmoplasia to be measured more accurately. Using image analysis, the amount of red colour, representing the desmoplastic tumour stroma, was calculated and expressed as the PD. As shown in the cumulative survival curve, patients with a PD $>4$ had a shorter survival than those with a PD $\leqslant 4$. Our results showed that the current semiquantitative scoring for desmoplasia is not a reliable method, because quantitative desmoplasia values were not significantly different among the different semiquantitative desmoplasia scores. Although univariate survival analysis showed both semiquantitative desmoplasia grades and PD to be relevant to prognosis, only the measurement of desmoplasia by the quantitative method retained a clear independent association with overall survival.

With a better understanding of the contribution of stromal tissue to cancer progression, new therapeutic agents might be targeted at inhibiting the crosstalk between cancer cells and the stroma. ${ }^{124}$ A potential target in anti-stroma treatment is transforming growth factor $\beta$, a well described cancer cell derived cytokine that activates the stroma and directly transdifferentiates fibroblasts into myofibroblasts. ${ }^{1}$ Other

\section{Take home messages}

- The percentage of desmoplasia (PD) measured by image analysis was found to be significantly associated with lymph vessel and venous invasion and was associated with survival-patients with PD $>4$ had a shorter survival than those with $P D \leqslant 4$

- Thus, PD measured by image analysis seems to be a significant prognostic indicator in patients with colorectal carcinoma and is a more accurate predictor than semiquantitative methods

- The improved method described in this study would be useful for routine prognostication examples include the induction of apoptosis in myofibroblasts, antiangiogenic factors, and matrix metalloproteinase inhibitors. ${ }^{1240}$ In addition, the extracellular matrix may contribute to the drug resistance of a solid tumour by preventing the penetration of therapeutic agents or reducing chemotherapy induced apoptosis in cancer cells. ${ }^{37}{ }^{38}$ Netti et al detected an extended collagen network in the more penetration resistant tumours, and identified collagen as a potential target of treatment to improve drug penetration. ${ }^{37}$

In our present study, the measurement of desmoplasia by computer assisted image analysis indicated that the amount of desmoplasia is an independent prognostic marker in primary colorectal carcinoma. With advances in our understanding of the contribution of the stroma to cancer progression, pathologists will probably be asked to assess the extent of desmoplasia in surgical pathology reports, for both prognostic and therapeutic purposes. Reliable, reproducible, and uncomplicated/easy to use methods for the measurement of desmoplasia, as proposed in our present study, should be established for further clinical trials.

\section{Authors' affiliations}

B Sis, S Sarioglu, A Kupelioglu, Department of Pathology, Dokuz Eylül University School of Medicine, Inciraltı, Izmir 35340, Turkey

S Sokmen, M Fuzun, Department of General Surgery, Dokuz Eylül University School of Medicine

M Sakar, Department of Biomedical Engineering, Dokuz Eylül University School of Medicine

\section{REFERENCES}

1 De Wever O, Mareel M. Role of tissue stroma in cancer cell invasion. J Pathol 2003;200:429-47.

2 Ohtani H. Stromal reaction in cancer tissue: pathophysiologic significance of the expression of matrix-degrading enzymes in relation to matrix turnover and immune/inflammatory reactions. Pathol Int 1998;48:1-9.

3 Hewitt RE, Powe DG, Carter Gl, et al. Desmoplasia and its relevance to colorectal tumour invasion. Int J Cancer 1993;53:62-9.

4 Lioatta LA, Kohn EC. The microenvironment of the tumour-host interface. Nature 2001;411:375-9.

5 Tuxhorn JA, McAlhany SJ, Dang TD, et al. Stromal cells promote angiogenesis and growth of human prostate tumours in a differential reactive stroma (DRS) xenograft model. Cancer Res 2002;62:3298-307.

6 Dingemans KP, Zeeman-Boeschoten IM, Keep RF, et al. Transplantation of colon carcinoma into granulation tissue induces an invasive morphotype. Int J Cancer 1993;54:1010-16.

7 Maeshima AM, Niki T, Maeshima A, et al. Modified scar grade. A prognostic indicator in small peripheral lung adenocarcinoma. Cancer 2002;95:2546-54.

8 Ronnov-Jessen L, Petersen OW, Bissell MJ. Cellular changes involved in conversion of normal to malignant breast: importance of the stromal reaction. Physiol Rev 1996:76:69-125.

9 Breuninger H, Schaumburg-Lever G, Holzschuh J, et al. Desmoplastic squamous cell carcinoma of skin and vermilion surface. Cancer 1997;79:915-19.

10 Compton CC, Fenoglio-Preiser CM, Pettigrew N, et al. American Joint Committee on Cancer prognostic factors consensus conference. Colorectal working group. Cancer 2000;88:1739-57.

11 Halvorsen TB, Seim E. Association between invasiveness, inflammatory reaction, desmoplasia and survival in colorectal cancer. J Clin Pathol 1989;42:162-6.

12 Compton CC, Fielding LP, Burgart $\sqcup$, et al. Prognostic factors in colorectal cancer. College of American Pathologists consensus statement 1999. Arch Pathol Lab Med 2000; 124:979-94.

13 Cohen AM, Kelsen D, Saltz L, et al. Adjuvant therapy for colorectal cancer. Curr Prob/ Surg 1997;34:611-12. 
14 Cooper HS. Intestinal neoplasias, In: Sternberg SS, Antonioli DA, eds. Diagnostic surgical pathology. Philadelphia: Lippincott Williams \& Wilkins, 1999: 1413-68.

15 Greene FL, Page DL, Fleming ID, eds. Colon and rectum. In: AJCC cancer staging handbook, Cancer staging manual. New-York: Springer-Verlag, 2002:113-24.

16 Bradbury P, Gordon KC. Connective tissues and stains. In: Bancroft JD, Stevens A, eds. Theory and practice of histological techniques. London: Churchill Livingstone, 1990:119-42.

17 Kavukcu S, Soylu A, Turkmen M, et al. Unilateral ureteroperitoneostomy in the management of hypoproteinemia in nephrotic rats with normal renal function. Tohoku J Exp Med 2003;201:67-73.

18 Demiral AN, Sarıoglu S, Birlik B, et al. Prognostic significance of EGF receptor expression in early glottic cancer. Auris Nasus Larynx 2004;31:417-24.

19 Grimm PC, Nickerson P, Gough J, et al. Computerized image analysis of Sirius red-stained renal allograft biopsies as a surrogate marker to predict long-term allograft function. J Am Soc Nephrol 2003;14:1662-8.

20 Masseroli M, O'Valle F, Andujar M, et al. Design and validation of a new image analysis method for automatic quantification of interstitial fibrosis and glomerular morphometry. Lab Invest 1998;78:511-22.

21 Zaitoun AM, Al Mardini H, Awad S, et al. Quantitative assessment of fibrosis and steatosis in liver biopsies from patients with chronic hepatitis C. J Clin Pathol 2001;54:461-5.

22 Barsky SH, Gopalakrishna R. Increased invasion and spontaneous metastasis of BL6 melanoma with inhibition of the desmoplastic response in $\mathrm{C} 57 \mathrm{BL} / 6$ mice. Cancer Res 1987;47:1663-75.

23 Peyrol S, Raccurt M, Gerard F, et al. Lysl oxidase gene expression in the stromal reaction to in situ and invasive ductal breast carcinoma. Am J Pathol 1997; 150:497-507

24 Quaranta V, Giannelli G. Cancer invasion: watch your neighbour hood. Tumori 2003;89:343-8.

25 Krtolica A, Campisi J. Cancer and aging: a model for the cancer promoting effects of the aging stroma. Int J Biochem Cell Biol 2002;34:1401-14.

26 Charous SJ, Stricklin GP, Netterville JL, et al. Expression of matrix metalloproteinases in head and neck squamous cell carcinoma. Ann Otol Rhinol Laryngol 1997;106:271-8.
27 Bosman FT, de Bruine A, Flohil C et al. Epithelial-stromal interactions in colon cancer. Int J Dev Biol 1993;37:203-11.

28 Martin M, Pujuguet $P$, Martin F. Role of stromal myofibroblasts infiltrating colon cancer in tumor invasion. Pathol Res Pract 1996;192:712-17.

29 Dimanche-Boitrel MT, Vakaet $L$, Pujuguet $P$, et al. In vivo and in vitro invasiveness of a rat colon-cancer cell line maintaining $\mathrm{E}$-cadherin expression: an enhancing role of tumor-associated myofibroblasts. Int J Cancer 1994;56:512-21

30 Lieubeau B, Heymann M, Henry F, et al. Immunomodulatory effects of tumorassociated fibroblasts in colorectal-tumor development. Int J Cancer 1999;81:629-36.

31 Colpaert $C$, Vermeulen $P$, van Beest $P$, et al. Intratumoral hypoxia resulting in the presence of a fibrotic focus is an independent predictor of early distant relapse in lymph node-negative breast cancer patients. Histopathology 2001;39:416-25.

32 Colpaert CG, Vermeulen PB, Fox SB, et al. The presence of a fibrotic focus in invasive breast carcinoma correlates with the expression of carbonic anhydrase IX and is a marker of hypoxia and poor prognosis. Breast Cancer Res Treat 2003;81:137-47.

33 Harris AL. Hypoxia-a key regulatory factor in tumour growth. Nat Rev Cancer 2002;2:38-47.

34 Cooper R, Sarioglu S, Sokmen S, et al. Glucose transporter-1 (GLUT-1): a potential marker of prognosis in rectal carcinoma? $\mathrm{Br} J$ Cancer 2003;89:870-6.

35 Sokmen S, Sarioglu S, Fuzun M, et al. Prognostic significance of angiogenesis in rectal cancer: a morphometric investigation. Anticancer Res 2001;21:4341-8

36 Caballero T, Perez-Milena A, Masseroli M, et al. Liver fibrosis assessment with semiquantitative indexes and image analysis quantification in sustainedresponder and non-responder interferon-treated patients with chronic hepatitis C. J Hepatol 2001;34:740-7.

37 Netti PA, Berk DA, Swartz MA, et al. Role of extracellular matrix assembly in interstitial transport in solid tumors. Cancer Res 2000;60:2497-503

38 Kouniavsky G, Khaikin M, Zvibel I, et al. Stromal extracellular matrix reduces chemotherapy-induced apoptosis in colon cancer cell lines. Clin Exp Metastasis 2002;19:55-60. 